\title{
Carta. Por un cambio en la evaluación de la investigación científica: "no tirar al niño junto con el agua sucia"
} Letter. For a change in the evaluation of scientific research: "don't throw the baby out with the bathwater"

\author{
Lluís Codina
}

Cómo citar esta carta:

Codina, Lluís (2021). “Carta. Por un cambio en la evaluación de la investigación científica: 'no tirar al niño junto con el agua sucia'". Profesional de la información, v. 30, n. 4, e300418.

https://doi.org/10.3145/epi.2021.jul.18

Carta recibida el 24-07-2021

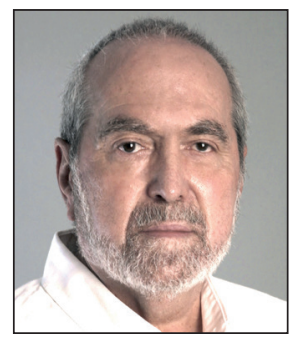

\author{
Lluís Codina $\triangle$ \\ https://orcid.org/0000-0001-7020-1631 \\ Universitat Pompeu Fabra \\ Departamento de Comunicación \\ Roc Boronat, 138, desp. 53.804 \\ 08018 Barcelona, España \\ Iluis.codina@upf.edu
}

\section{Resumen}

Respuesta a dos cartas recientes publicadas en la revista Profesional de la información sobre la necesidad de cambios en la evaluación de la ciencia. Se proponen dos elementos que deberían formar parte de cualquier futuro modelo de evaluación, a saber: la obligación del rendimiento de cuentas mediante evaluaciones externas, y el papel central de la comunicación de la ciencia. Así mismo, se presentan ejemplos de cambios en profundidad que pueden acometerse y que respetan estos dos principios esenciales. Adicionalmente se propone una revisión de conceptos a veces mal interpretados, como la expresión publish or perish, porque enmascara el auténtico problema en lugar de denunciarlo, o el de autoplagio porque no solamente es un oxímoron sino que sitúa el plagio en un terreno de prácticas aceptables, lo que carece de sentido.

\section{Palabras clave}

Evaluación de la ciencia; Evaluación de la investigación; Rendimiento de cuentas; Comunicación académica; Publish or perish; Autoplagio; Contenido reciclado.

\begin{abstract}
This is a response to two letters published recently in the Profesional de la información journal on the need for changes in the evaluation of science. Two elements are proposed that should be part of any future evaluation model, namely the obligation for accountability through external evaluations, and the central role of science communication. Likewise, examples of in-depth changes that can be undertaken and that respect these two essential principles are presented. Additionally, a review of sometimes misinterpreted concepts is proposed, such as the expression "publish or perish," because it masks the real problem instead of denouncing it, or that of self-plagiarism because not only is it an oxymoron but it also places plagiarism in a field of acceptable practices, which is meaningless.
\end{abstract}

\section{Keywords}

Science assessment; Research evaluation; Accountability; Scholarly communication; Publish or perish; Self-plagiarism; Text recycling. 


\section{Introducción}

En los últimos años, y con razón, abundan los llamamientos a un cambio en la evaluación de la ciencia. Más apropiadamente, en la forma en la que se evalúan las contribuciones científicas y las carreras académicas por parte de agencias de evaluación y de otros actores del mundo científico.

Estas evaluaciones tienen un impacto directo en la promoción personal de los científicos y en el progreso de su carrera académica, así que no es extraño que, sea cual sea el procedimiento de evaluación, siempre cause controversia.

Una muestra de este movimiento a favor del cambio son las cartas recientes publicadas en esta revista Profesional de la información (EPI) por autores tan relevantes como Emilio Delgado, Ernest Abadal, Ismael Ràfols (Delgado et al., 2021) por un lado e Isidro Aguillo (Aguillo, 2021) por otro. Estas cartas han motivado esta que tiene el lector delante. La diferencia es que mi interés en este caso no es solamente aportar ideas de ruptura, sino también defender las cosas que creo que merecen preservarse, así como presentar ejemplos de opciones alternativas.
Hay dos principios que cualquier modelo de evaluación de la ciencia debe considerar: la idea del rendimiento de cuentas con evaluaciones externas al que están obligadas las actividades financiadas con capital público, y la del papel central de la comunicación científica en la actividad científica

Ciertamente, la forma que adopten las nuevas evaluaciones no puede deberse a ninguna propuesta individual. Por el contrario, deben ser fruto de un trabajo de estudio profundo y racional, a cargo de un equipo solvente que trabaje bajo condiciones de publicidad y transparencia. Pero mientras tanto, debemos evitar ofrecer elementos de demolición sin ofrecer al menos, un esbozo de cómo podrían ser los de construcción.

Con estos antecedentes, me voy a centrar en dos cuestiones, para luego dar algunas ideas de cómo podrían ser los cambios en la evaluación teniendo en cuenta estas mismas cuestiones. Los dos puntos son los siguientes:

- La necesidad de las evaluaciones por motivos elementales de rendición de cuentas.

- La racionalidad de considerar la comunicación académica, no como el único, pero sí como uno de los factores principales en cualquier modelo de evaluación de la actividad científica.

Una vez examinados estos dos puntos, presentaremos un tercero sobre el posible margen de que disponemos en caso de aceptar estas dos premisas.

\section{Responsabilidad social y rendimiento de cuentas}

La premisa importante aquí es que el mundo científico/académico está financiado totalmente o en parte por capital público. Es el caso de los proyectos de investigación que reciben fondos estatales o de la Unión Europea. Es el caso también de los sueldos de profesores de universidad, en especial, de las universidades públicas, aunque las universidades privadas también se benefician, con todo merecimiento, de subvenciones.

De todos modos, las actividades que desarrollan las universidades son tan decisivas para la sociedad, que deben estar sometidas a rendimiento de cuentas, incluso si fuera el caso, si me permiten la expresión absurda, de que el dinero les cayera del cielo.

Ahora bien, el punto importante aquí es que todo rendimiento de cuentas debe tener una parte de evaluación externa. Es cierto que las autoevaluaciones son necesarias, pero son inútiles sin un componente de evaluación externa.

\section{La publicaciones evaluadas como uno de los elementos nucleares}

La segunda idea que todo debate sobre el cambio, por radical que sea, debe tener presente es la siguiente: una investigación que no se publica, no existe. Por tanto, no solamente no es irracional que las evaluaciones se basen en las publicaciones, sino que es una exigencia para cualquier posible modelo de evaluación.

Además, ya hemos dicho que en toda evaluación, para merecer este nombre, la de tipo externo es imprescindible. Son valiosas las publicaciones que los propios autores pueden hacer bajo la forma de preprints, working papers, informes autoeditados, etc. Pero es imprescindible que una parte de sus publicaciones sea el resultado del exigente escrutinio obtenido mediante el denominado peer review, clave de bóveda del sistema de comunicación de la ciencia.

\section{4. ¿Dónde podemos situar elementos de cambio?}

En lo que sigue, sin pretensiones de exhaustividad, propongo algunos puntos que pueden ser objeto de renovación, más o menos profunda según los casos, y que respetan los principios señalados:

\section{Producciones no bibliográficas}

Deben aceptarse formas de producción distintas de las bibliográficas. Y cada disciplina debería tener capacidad para definirlas a partir de propuestas razonadas. Para poner dos ejemplos fáciles: en ciencias de la computación, podría aceptarse la producción de software. En bellas artes, podrían aceptarse producciones artísticas. En todos los casos, fueran como fueran las clases de producción aceptables (y no debería haber una lista cerrada), las condiciones elementales para su aceptación 
es que sean susceptibles de evaluación externa. Para seguir con los ejemplos, para aceptar un nuevo software, su autor podría aportar pruebas de su superior rendimiento comparado con otros, o dictámenes externos por parte de colegas o empresas que lo hayan utilizado. Para la producción artística, su autor podría aportar documentación sobre un proceso creativo resultado de la investigación, junto con críticas de expertos en arte o testimonios de que su obra ha sido aceptada en galerías o exhibiciones. Son solo ejemplos, y puede que no muy afortunados, pero creo que cumplen la misión de ilustrar de qué manera cualquier clase de producción no bibliográfica puede ser objeto de evaluación externa. Seguro que los expertos en ciencias de la computación y en bellas artes son capaces de diseñar procesos mucho más sofisticados y oportunos que estos torpes ejemplos.

\section{Publicaciones distintas de los artículos de revistas}

Actualmente los artículos de revista tienen un peso que en algunas áreas es excesivo o incluso injustificado. Para determinadas áreas, o para aquellas que así lo reclamen, otros vectores para comunicar la ciencia deberían ser aceptados (libros, p. ej.), y no como una contribución menor, sino en pie de igualdad, o superior al de los artículos. Como en el caso anterior, la clave está en demostrar que se trata de publicaciones que han tenido algún tipo de evaluación externa. En el caso de los libros (o capítulos de libro), hay muchas editoriales que aceptan propuestas de manuscritos solo después de algún proceso de selección de calidad. Lo mismo para las comunicaciones presentadas en congresos. Otros criterios podrían ser el prestigio académico de las editoriales, y de los congresos su carácter internacional, etc.

\section{Evaluaciones al nivel de la investigación}

No puede volver a darse, de ningún modo, que un investigador vea rechazada su solicitud para el reconocimiento de su investigación (sea un sexenio o la forma de evaluación de su actividad científica que se fije en un futuro) o para una acreditación por no aportar artículos en revistas de primer cuartil. Esto debería considerarse una aberración. Los factores de impacto o los cuartiles de las revistas pueden invocarse como un elemento secundario o terciario, y siempre indirecto de calidad, puesto que se sitúan en el nivel de la revista, no del artículo. La investigación, como reclaman DORA y Leiden, debe ser evaluada en sí misma y con indicadores de su propio nivel. Para ello, pueden utilizarse elementos tales como un impact statement que ponga el énfasis en el impacto social, las citas recibidas y el propio juicio de los expertos responsables de la evaluación, por mencionar algunos. En un contexto evaluador así, el factor de impacto de la revista, cuando sea favorable puede utilizarse como factor positivo adicional, aunque sea de segundo o tercer nivel. En cambio, debe quedar completamente excluido el uso de cuartiles como factor negativo en cualquier caso.

\section{Valorar el impacto social y no solo el académico}

El uso (inadecuado) de indicadores de impacto, tales como los cuartiles de las revistas, señala un foco exclusivo en el impacto académico. Sin embargo, una ciencia financiada principalmente con capital público exige la aplicación de indicadores de impacto social. Mientras que el impacto académico es relativamente fácil de determinar, el impacto social es mucho más difícil, pero es imperioso hacer esfuerzos para identificar y establecer formas de medirlo. Sería una forma de promover una ciencia responsable que se preocupe más de su impacto en el bienestar de las personas y de la sociedad en su conjunto, que su impacto en otros colegas.

\section{A favor de DORA y Leiden, pero en todas sus dimensiones}

Cada vez estoy más convencido que la clave para cualquier cambio en los procesos de evaluación pasa por una lectura atenta e integral de las declaraciones denominadas DORA (DORA, s.f.) y Leiden (Hicks et al., 2015), que son las que han inspirado buena parte de los puntos anteriores.

Lo digo porque he visto rechazar algunos de los puntos que presentamos aquí invocando DORA o Leiden, cuando no es esto lo que dicen. Si queremos invocar DORA o Leiden, hay que considerarlas en su integridad, no solo en la parte que nos interesa.

\section{Bonus: dos tópicos que necesitan revisión}

Para concluir, dos tópicos muy mal digeridos, a los que me gustaría referirme con ocasión de esta carta. Uno es la famosa expresión publish o perish! y el otro el desafortunado término "autoplagio". Veamos:

\section{Publish o perish!}

Como supuesto retrato de la situación actual envía un mensaje equivocado, trasladando a los ciudadanos una idea falaz. Un caso significativo: para obtener un sexenio de investigación, solamente pueden presentarse a evaluación 5 contribuciones, esto es imenos de una por año! Otro ejemplo: hasta donde yo conozco, en muchas universidades y departamentos hay profesores permanentes o funcionarios que no publican nunca o casi nun-
La frase publish or perish! en lugar de denunciar un supuesto problema real, lo enmascara y traslada una idea equivocada a los ciudadanos. El problema real, como denuncian DORA y Leiden, es el uso totalmente inadecuado de indicadores, aunque tampoco es el único 
ca. No solamente siguen en sus puestos sin que nadie les moleste (como debe ser), sino que siguen progresando académicamente, lo que me parece muy bien, porque en las universidades necesitamos todos los perfiles de profesorado. Si nos tomásemos en serio lo de publish or perish habría que reportar despidos más o menos de forma continua por este motivo. En lugar de esto, progresan en cargos de gestión y docentes, por lo que ven mejorado su salario de forma progresiva en base a las evaluaciones quinquenales de docencia, a lo que suman los complementos por cargos de gestión, a lo que hay que sumar aún los trienios. Todo lo cual, ya he señalado que me parece perfecto. La razón es que necesitamos todos los perfiles en la universidad y algunos están más orientados a la docencia o la gestión, que a la investigación, mientras que es muy raro que un profesor sea muy bueno en los tres perfiles a la vez. Además, últimamente ha aparecido la figura de los "sexenios de transferencia", que dignifican formas de aportaciones científicas a la sociedad distintas de la publicación. En este contexto, la expresión publish or perish! solamente consigue enmascarar el problema real , que es el uso inadecuado del factor de impacto..

\section{Autoplagio}

Es un término que debemos desterrar de nuestro lenguaje por razones de ética. En primer lugar, el plagio es rechazable en cualquier grado y carece de umbrales de tolerancia. En cambio, el uso de contenidos del mismo autor en más de una de sus publicaciones es algo sometido a consideración. Hay usos aceptables de contenido reciclado, tanto desde el punto de vista ético como legal como argumenta en un detallado estudio Moskovitz

El autoplagio es un oxímoron que, además, blanquea en parte al plagio. En su lugar, los expertos recomiendan el uso de text recycling que puede tener, a diferencia del plagio, usos aceptables tanto ética como legalmente

(2021), cosa que no ocurre con el plagio. Luego, el término que debemos usar es el de texto o contenido "reciclado", tal como recomiendan los expertos (Moskovitz, 2021; BioMed Central; COPE, s.f.) nunca el de autoplagio que además es un oxímoron. A partir de aquí, su aceptación (o no) dependerá de consideraciones diversas.

\section{A modo de conclusión}

La evaluación de la ciencia necesita cambios profundos que no pueden proceder de propuestas individuales como esta, sino del trabajo de un equipo solvente que sea elegido, y que trabaje en base a criterios de eficiencia, transparencia y publicidad. Y no sin escuchar a las partes afectadas.

Estos cambios pueden tener un alcance enorme: algunas de las consecuencias son susceptibles de aplicarse de manera inmediata con casi total seguridad (p. ej. las que afectan a los cuartiles y los tipos de publicaciones aceptables). Pero se han presentado dos principios que, no solo parecen necesarios en cualquier modelo, sino que seguramente pueden considerarse una precondición necesaria para la legitimidad y racionalidad de cualquiera de ellos.

No puedo dejar esta carta sin expresar mi reconocimiento a algunos de los investigadores españoles de los que más he aprendido en este ámbito. Son autores que sigo desde hace muchos años, y de todos puedo decir que sus aportaciones han sido decisivas para mi formación.

Se trata, entre otros, de Ernest Abadal, Emilio Delgado e Isidro Aguillo. Si tengo la suerte de que lean esta carta, seguramente estarán en desacuerdo con parte de lo que he expuesto, sino con todo. Por tanto, esto no es un intento de endorsarles ninguna responsabilidad: mientras los posibles aciertos les deben mucho, los errores son solo míos.

\section{Referencias}

Aguillo, Isidro F. (2021). “Respuesta a Delgado-López-Cózar et al. Factor de impacto: Muchas declaraciones y pocos resultados. Letter". Profesional de la información, v. 30, n. 3, e300315.

https://doi.org/10.3145/epi.2021.may.15

BioMed Central; COPE (s. f.). Text recycling guidelines. https://publicationethics.org/files/Web_A29298_COPE_Text_Recycling.pdf

Delgado-López-Cózar, Emilio; Ràfols, Ismael; Abadal, Ernest (2021). “Letter: A call for a radical change in research evaluation in Spain". Profesional de la información, v. 30, n. 3, e300309.

https://doi.org/10.3145/epi.2021.may.09

DORA. San Francisco Declaration on Research Assessment (2012). https://sfdora.org/read

Hicks, Diana; Wouters, Paul; Waltman, Ludo; De-Rijcke; Ràfols, Ismael (2015). “Bibliometrics: The Leiden Manifesto for research metrics". Nature, n. 520, pp. 429-431.

https://doi.org/10.1038/520429a

Moskovitz, Cary (2021) "Standardizing terminology for text recycling in research writing". Learned publishing, v. 34, n. 3, pp. 370-378.

https://doi.org/10.1002/leap.1372 